\title{
Diagnosis and definition of severe refractory asthma: an international consensus statement from the Innovative Medicine Initiative (IMI)
}

\author{
Elisabeth H Bel, ${ }^{1}$ Ana Sousa, ${ }^{2}$ Louise Fleming, ${ }^{3}$ Andrew Bush, ${ }^{4} \mathrm{~K}$ Fan Chung, ${ }^{5}$ \\ Jennifer Versnel, ${ }^{6}$ Ariane H Wagener, ${ }^{1}$ Scott S Wagers, ${ }^{7}$ Peter J Sterk, ${ }^{1}$ \\ Chris $\mathrm{H}$ Compton, ${ }^{8}$ on behalf of the members of the Unbiased Biomarkers for the \\ Prediction of Respiratory Disease Outcome (U-BIOPRED) Consortium, Consensus \\ Generation $^{9}$
}

${ }^{1}$ Department of Respiratory Medicine, Academic Medical Centre, University of

Amsterdam, Amsterdam, The Netherlands

${ }^{2}$ GlaxoSmithKline, Stevenage, UK

${ }^{3}$ Clinical Trials and Evaluation Unit, Imperial College London, London, UK

${ }^{4}$ Department of Paediatric Respiratory Medicine, Royal Brompton Hospital, London, UK ${ }^{5}$ Airways Disease, National Heart and Lung Institute, Imperial College, London, UK ${ }^{6}$ Asthma UK, London, UK ${ }^{7}$ BioSci Consulting, Maasmechelen, Belgium ${ }^{8}$ European Federation of Pharmaceutical Industries and Associations (EFPIA), EFPIA Headquarters, Brussels, Belgium ${ }^{9}$ European Union (EU) Innovative Medicines Initiative (IMI) Program Understanding Severe Asthma, Consortium Unbiased Biomarkers for the Prediction of Respiratory Disease Outcomes (U-BIOPRED)

\section{Correspondence to}

Professor Dr Elisabeth $\mathrm{H}$ Bel,

Department of Respiratory

Medicine, Academic Medical

Centre, University of

Amsterdam, Meibergdreef 9 ,

1105 AZ Amsterdam, The

Netherlands;

e.h.bel@amc.uva.n

For author footnote see end of the article.

Received 17 October 2010 Accepted 20 October 2010 Published Online First 23 November 2010

\section{ABSTRACT}

Patients with severe refractory asthma pose a major healthcare problem. Over the last decade it has become increasingly clear that, for the development of new targeted therapies, there is an urgent need for further characterisation and classification of these patients. The Unbiased Biomarkers for the Prediction of Respiratory Disease Outcomes (U-BIOPRED) consortium is a pan-European public-private collaboration funded by the European Commission Innovative Medicines Initiative of the European Union. U-BIOPRED aims to subphenotype patients with severe refractory asthma by using an innovative systems biology approach. This paper presents the U-BIOPRED international consensus on the definition and diagnosis of severe asthma, aligning the latest concepts in adults as well as in children. The consensus is based on existing recommendations up to 2010 and will be used for the selection of patients for the upcoming U-BIOPRED study. It includes the differentiation between 'problematic', 'difficult' and 'severe refractory' asthma, and provides a systematic algorithmic approach to the evaluation of patients presenting with chronic severe asthma symptoms for use in clinical research and specialised care.

\section{INTRODUCTION}

Since the last international consensus meetings in 1999 (European Respiratory Society (ERS) Task Force ${ }^{1}$ ) and 2000 (American Thoracic Society (ATS) Workshop ${ }^{2}$ ), novel insights into an accurate definition of severe asthma have emerged. Defining and phenotyping of patients with severe asthma is becoming increasingly important for the development of new therapies, ${ }^{3-6}$ as some patients who were previously classified as having severe asthma because of poor asthma control despite maximal doses of conventional therapy may eventually become well controlled with a targeted phenotype-specific treatment. ${ }^{7-14}$ In addition, it seems appropriate to differentiate between 'difficult-to-treat' and 'severe refractory' asthma, since these subtypes of asthma may all present with severe symptoms but could represent different conditions or phenotypes with disparate underlying causes that would benefit from targeted therapy.

\section{DIAGNOSIS AND DEFINITION OF SEVERE ASTHMA OVER THE LAST 15 YEARS}

Various documents proposing different clinical definitions of 'severe asthma' in adults and children have been published over the last 15 years by international task forces, workshops, networks and guideline committees.

\section{Adult guidelines}

In the Global Initiative for Asthma ${ }^{15} 1995$ and 2002 updates and the National Asthma Education and Prevention Programme $1997^{16}$ guidelines, overall asthma severity was primarily based on the patient's clinical characteristics prior to commencing treatment. Off-treatment severity was classified into intermittent, mild persistent, moderate persistent and severe persistent, based on symptoms, short-acting $\beta_{2}$ agonist use, night time awakening and peak expiratory flow or the percentage predicted forced expiratory volume in $1 \mathrm{~s}\left(\mathrm{FEV}_{1}\right)$. This initial classification was used to determine the patient's initial treatment but did not take into account disease responsiveness to treatment.

In 1999 an ERS Task Force ${ }^{1}$ defined 'difficult/ therapy-resistant asthma' as poorly controlled asthma and a continued requirement for shortacting $\beta_{2}$ agonists despite delivery of a reasonable dose of inhaled corticosteroids (ICS) and follow-up by a respiratory specialist for a period of $>6$ months. During this period, asthma management had to be carried out according to published asthma guidelines.

In 2000 an ATS Workshop ${ }^{2}$ adopted the term 'refractory asthma' and developed a definition by consensus. The definition included one of two major criteria (continuous high-dose ICS or oral corticosteroids for $>50 \%$ of the time during the previous year), with two out of seven additional minor criteria: requirement of additional controller medications, aspects of disease stability, exacerbations and lung function. This definition was adopted by the NIH/NHLBI-sponsored Severe Asthma Research Program (SARP) ${ }^{17}$ network.

The European Network for Understanding Mechanisms of Severe Asthma ${ }^{18}$ defined 'severe asthma' in 2003 as confirmed asthma (typical asthma symptoms, reversibility in $\mathrm{FEV}_{1}$ or airway hyper-responsiveness) plus the occurrence of one or 
more exacerbations in the previous year despite oral corticosteroids or high-dose ICS. ${ }^{18}$ The Epidemiology and Natural History of Asthma: Outcomes and Treatment Regimens (TENOR) study group ${ }^{19}$ included patients with high use of the healthcare system or high medication use in the past year.

In 2007 an international workshop was organised in Paris to discuss the important questions in severe asthma. ${ }^{20}$ This workshop agreed that a diagnosis of 'severe asthma' should be reserved for those patients who have refractory asthma after an extensive re-evaluation of the correct diagnosis, aggravating comorbidities and environmental factors and an appropriate observation period of at least 6 months.

\section{Paediatric guidelines}

In children there has been a lack of general consensus on the definition of severe asthma. In 2008 an ERS Task Force on Definition, Assessment and Treatment of Wheezing Disorders in Preschool Children ${ }^{21}$ stated that making a diagnosis of asthma in preschool children is unfeasible. Whereas in adults and children $>6$ years of age there is consensus that asthma is characterised by airway inflammation, ${ }^{15}$ this has been poorly studied in preschool children, ${ }^{22}$ and may be absent in very young children who wheeze. ${ }^{23}$ The Task Force members therefore adopted a symptoms-only descriptive approach for children $<6$ years of age, and used the terms 'episodic (viral) wheeze' to describe children who wheeze intermittently and are well between episodes and 'multiple-trigger wheeze' for children who wheeze both during and outside discrete episodes. ${ }^{21}$

In 2008 the Problematic Severe Asthma in Childhood Initiative (PSACI) group ${ }^{24}$ proposed the use of the term 'problematic severe asthma' to describe all school-aged children who, despite regular treatment with $\geq 800 \mu \mathrm{g} /$ day budesonide or equivalent of ICS plus a long-acting $\beta$-agonist, a leukotriene receptor antagonist or theophylline, have poorly controlled asthma-that is, daily asthma symptoms, recurrent severe asthma exacerbations (or a single near-fatal asthma attack), persistent airflow obstruction or the necessity for the prescription of chronic oral steroids to achieve control of asthma. 'Difficult-to-treat asthma' was defined as asthma where the poor control is due to a wrong diagnosis or comorbidities, the inability and unwillingness to adhere to the prescribed treatment regimens or adverse psychological and environmental factors. 'Severe therapy-resistant asthma' was defined by the same group as 'difficult' asthma that remains uncontrolled despite attention to and resolution of all these factors.

\section{SHORTCOMINGS OF PREVIOUS DEFINITIONS ON SEVERE ASTHMA}

When studying the above-mentioned definitions of 'severe asthma', it appears that they have been refined and sharpened over the years. After the initial Global Initiative for Asthma $2005^{15}$ and National Asthma Education and Prevention Programme $1997^{16}$ guidelines in which overall asthma severity was based on the patient's clinical characteristics prior to commencing treatment, an assessment of asthma severity in patients on treatment seemed to be necessary. ${ }^{25}$

The definition of 'difficult/therapy-resistant asthma' by the ERS Task Force in $1999^{1}$ described patients with poorly controlled asthma despite prescription of a reasonable dose of ICS (defined as $\geq 2000 \mu \mathrm{g}$ beclometasone or the equivalent dose in adults and $\geq 800 \mu \mathrm{g}$ beclometasone or equivalent), and emphasised the need for addressing (1) the diagnosis of asthma; (2) adequate management of asthma; (3) compliance with treatment; (4) identification of exacerbating factors; and (5) exclusion of other diagnoses. Care of the patient by a respiratory specialist for at least 6 months was advisable. The proposed definition of the ERS Task Force was inclusive with the recognition that difficult/therapy-resistant asthma could be due to poor adherence, incorrect inhaler technique, psychological problems and comorbidities. It was also recognised that the definition could be adjusted according to the objectives of any individual research project.

The criteria for 'refractory asthma' as determined by the ATS Workshop in $2000^{2}$ were more strictly defined in terms of the criteria of severity of asthma ( $\geq 2$ of 7 criteria) in patients who were on high-dose ICS and/or oral corticosteroids for $>50 \%$ of the time. Moore and colleagues ${ }^{17}$ in their 2007 report on patients with severe asthma in the Severe Asthma Research Project (SARP) using the definition of the ATS Workshop found that the factors that best discriminated mild/moderate from severe asthma (apart from the use of high-dose ICS or oral corticosteroids $>50 \%$ of the time) were the use of multiple controller medications (including long-acting $\beta_{2}$ agonists), $\geq 3$ bursts of oral corticosteroids in the previous year or a history of at least one severe exacerbation requiring hospitalisation during the last year.

The most recent definitions of severe therapy-resistant asthma are the ones proposed by the Paris Workshop in 2007 for adults $^{20}$ and the PSACI group in 2009 for children. ${ }^{26}$ These definitions distinguished patients with 'severe refractory asthma' from those with 'difficult-to-treat asthma', the latter presenting with uncontrolled asthma due to other factors than asthma itself, including persistent environmental exposures, aggravating comorbidities, poor adherence and inadequate inhalation technique. This distinction is important because patients with difficult-to-treat asthma may not be candidates for immune suppressive or innovative anti-inflammatory therapies.

Finally, in April 2009 the WHO Consultation on Severe Asthma proposed a global definition of asthma severity which should be applicable in most circumstances in low-, middle- and high-income countries. ${ }^{27}$ Severe asthma was defined as 'uncontrolled asthma which can result in risk of frequent severe exacerbations (or death) and/or adverse reactions to medications and/or chronic morbidity (including impaired lung function or reduced lung growth in children)'. The WHO Consultation adopted the definitions of 'severe' and 'difficult' asthma from the Paris Workshop in $2007^{16}$ and extended it with a third group of patients with 'untreated' severe asthma. The latter group is, of course, of major importance in low-income countries where asthma drugs are not readily available to everyone and asthma deaths are still occurring.

\section{APPROACH TO EVALUATING PATIENTS WITH SEVERE ASTHMA SYMPTOMS}

For a correct diagnosis of severe refractory asthma, it is mandatory that patients who present with severe asthma symptoms or recurrent exacerbations are evaluated in a stepwise manner to address the following issues (figure 1).

\section{Distinction between severe and uncontrolled asthma}

Severe asthma should be distinguished from uncontrolled asthma. Uncontrolled asthma refers to the extent to which the manifestations of asthma have not been reduced or removed by treatment. $^{28}$ Asthma control incorporates components of current clinical control including symptoms, use of rescue 
Figure 1 Algorithm to diagnose a patient with severe refractory asthma. NSAID, non-steroidal anti-inflammatory drug; PEF, peak expiratory flow.

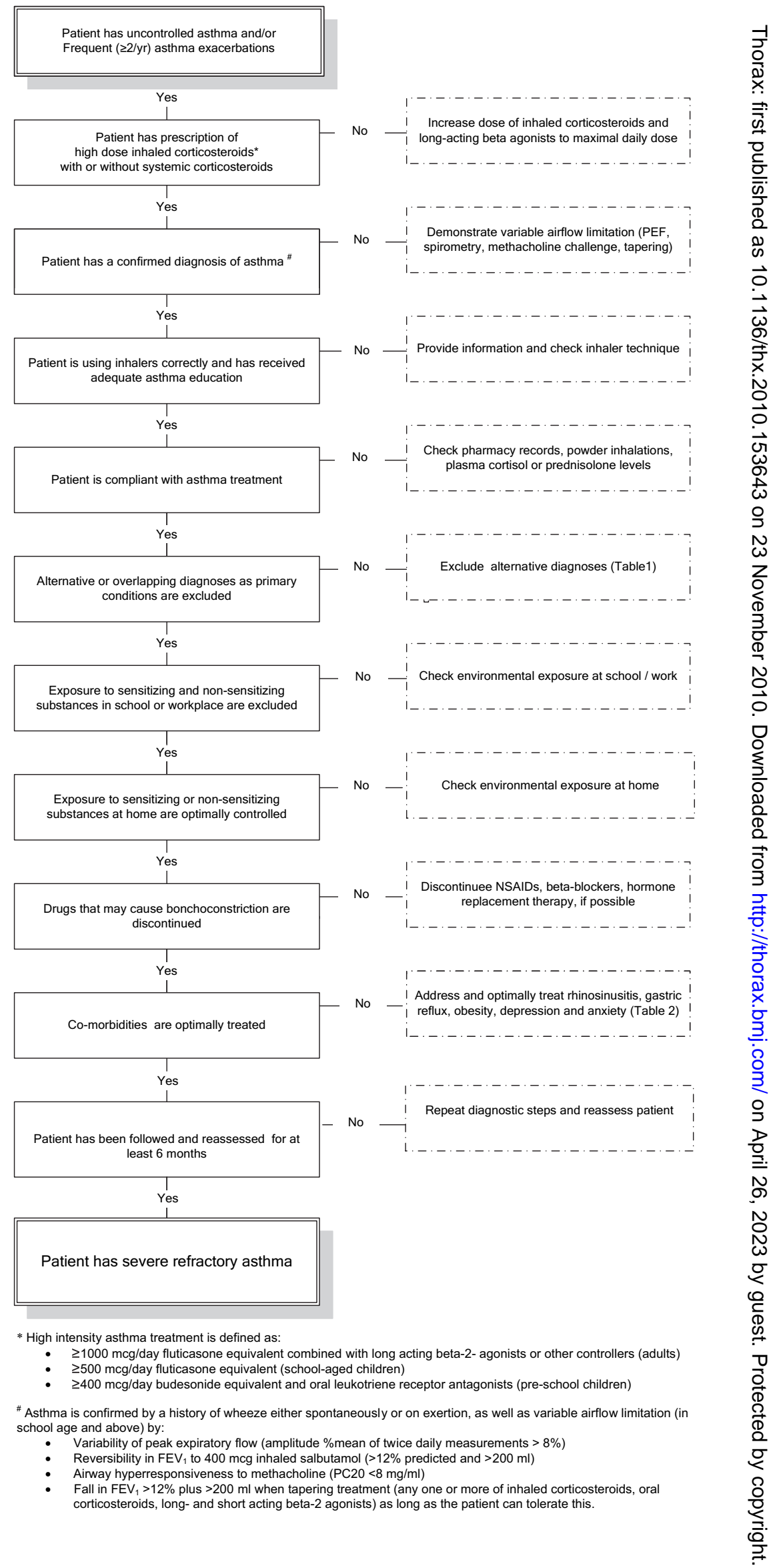


medication and lung function, as well as future risks. Asthma severity is determined by the intensity and phenotype of the underlying disease, both of which may be characterised by pathological and physiological markers. These markers can also be used to estimate future risk of exacerbation or decline in lung function.

Exacerbations are a prominent feature of both poorly controlled and severe asthma. Improving baseline asthma control with ICS can reduce the risk of exacerbations in patients with atopic asthma, ${ }^{29}$ but control of daily symptoms does not always imply control of exacerbations. ${ }^{30}$ Baseline disease control and exacerbations are most probably driven by different factors.

\section{Adherence to high-intensity asthma treatment}

A significant proportion of patients with uncontrolled asthma who are prescribed high doses of ICS do not take their medicines. In a case series, $50 \%$ of patients prescribed oral steroids were found to be non-adherent when assessed by plasma prednisone and cortisol concentrations. ${ }^{31}$ Also, other studies in adults and children in the USA and the UK showed that overall adherence to ICS was approximately $50 \% .^{32} 33$ Adherence to ICS was significantly and negatively correlated with the number of emergency department visits, the number of fills of an oral steroid and the total days' supply of oral steroid. Eight per cent of patients never filled their ICS prescription. ${ }^{34}$ Thus, despite persistent symptoms, many patients choose not to take their prescribed treatment, mainly because they perceive it to be unnecessary, too complex, too expensive in some healthcare systems or because they are concerned about potential adverse effects. ${ }^{35}$ In the investigation of patients presenting with severe asthma, it is therefore critical to check adherence, either by measuring serum cortisol, prednisolone and theophylline levels where appropriate, making home visits or checking lists of prescriptions from pharmacies. The Medication Adherence Rating Scale (MARS), a questionnaire that has been developed to estimate patient adherence with treatment, ${ }^{36}$ may be a helpful instrument but needs further validation.

\section{Establishing a secure diagnosis of asthma}

There are many conditions that may mimic severe refractory asthma, both in children and in adults. Since these conditions do not respond to high-intensity asthma treatment, they may easily be mistaken for severe asthma. A list of common alternative diagnoses and how they should be diagnosed is given in table 1.

Every patient with asthma who does not seem to respond to high-intensity asthma treatment should undergo objective tests to confirm the diagnosis of asthma. This includes tests to demonstrate variable airflow limitation such as daily peak expiratory flow measurements, reversibility tests with a bronchodilator drug, challenge tests with a bronchoconstricting agent or a steroid-tapering trial. Although there is no definitive diagnostic test for asthma, the repeated failure to demonstrate variable airflow obstruction over time, with treatment or under bronchial provocation tests should seriously call into question the diagnosis of asthma.

\section{Addressing and treating aggravating factors and comorbidities}

Mild to moderate asthma can become severe by the influence of exogenous or endogenous aggravating factors. ${ }^{37}$ These factors can be either trigger factors, coexisting conditions or part of the asthma syndrome itself.

\section{Trigger factors}

Trigger factors include (often concealed) indoor allergens, environmental pollutants, toxic fumes, occupational agents or drugs that can provoke asthma attacks. Several studies have shown that adults and children with severe allergic asthma are exposed to higher levels of allergens at home $e^{38}$ or at school ${ }^{39}$ to which they are sensitised, compared with subjects with mild asthma. Many patients with severe asthma are sensitised to fungi such as Aspergillus spp., Cladosporium spp., Alternaria spp., Penicillium spp., Candida spp., Trichophyton spp. and others. In these patients, antifungal therapy may lead to significant clinical improvement. ${ }^{40}$ Unfortunately there is no consensus yet on how to identify relevant environmental triggers.

Patients with asthma who smoke have more severe symptoms, reduced sensitivity to ICS ${ }^{41}$ and are more likely to be admitted to hospital for asthma than non-smokers with asthma. ${ }^{42}$ Smoking cessation improves asthma control, ${ }^{43}$ although complete recovery of steroid sensitivity does not occur in most patients. ${ }^{44}$ For children whose asthma is triggered by smoke exposure from their parents, ${ }^{45}$ effective treatment of the child's asthma must include smoking cessation for the child's parents.

Many occupational agents may aggravate asthma in adults. More than 200 high and low molecular agents have been identified that can induce work-related asthma or aggravate preexisting mild to moderate asthma. ${ }^{46} 47$

In some patients, asthma severity may be related to specific drugs such as $\beta$ blockers, ACE inhibitors or aspirin. Non-selective $\beta$-blockers ${ }^{48}$ and ACE inhibitors ${ }^{49}$ can induce significant bronchoconstriction in patients with asthma after acute dosing. Asthma that is exacerbated by aspirin is usually severe and, in half the patients, adequate control of asthma can only be achieved with oral corticosteroids. ${ }^{50}$ Removal of all of these drugs may substantially ameliorate asthma, although this is not always the case with aspirin-induced asthma. In this case, aspirin desensitisation might be useful. ${ }^{51}$

\section{Coexisting conditions (comorbidities)}

Disorders that often coexist with severe asthma and may make asthma worse include reflux disease ${ }^{52}$ and obesity (table 2). ${ }^{53}$ The exact relationship between gastro-oesophageal reflux and severe asthma has not been fully established, but many studies suggest that there is an interaction in the pathophysiology between the two disease processes. A correlation between gastro-oesophageal reflux and worsening of respiratory symptoms in patients with severe asthma has been convincingly shown, ${ }^{54}$ but the benefits of antireflux therapy are disappointing. ${ }^{52}$ It has been suggested that, in this subset of patients, fundoplication may be efficacious. ${ }^{55}$

Asthma and obesity are frequently associated, but the contribution of obesity to 'difficult-to-treat' asthma and the mechanisms responsible for this relationship have not been fully clarified. Bariatric surgery may lead to substantial weight loss and is associated with substantially decreased asthma symptoms ${ }^{56}$ However, the evidence that weight control interventions are associated with improvements in asthma control remains controversial. $^{57} 58$

\section{Conditions that are part of the severe asthma syndrome}

Asthma and rhinosinusitis often coexist and are believed to represent a spectrum of the same disease entity. In particular, in adults, chronic rhinosinusitis and nasal polyps have been shown to be important components of severe steroid-dependent asthma. ${ }^{59}$ Nasal symptoms and CT imaging of sinonasal involvement are related to asthma severity, sputum eosinophil 
Table 1 Tests to distinguish severe asthma from alternative diagnosis that may mimic asthma

\begin{tabular}{|c|c|}
\hline Routine screening test in adults & Exclusion (if test is normal) \\
\hline Air trapping measured by body plethysmography & Bronchiolitis obliterans \\
\hline Carbon monoxide diffusion capacity & Emphysema or parenchymal lung disease \\
\hline \multirow[t]{4}{*}{ Chest HRCT scan } & Parenchymal lung disease \\
\hline & Bronchiolitis obliterans \\
\hline & Bronchiectasis \\
\hline & Congestive heart failure \\
\hline D-dimer & Recurrent pulmonary embolism \\
\hline Suspected alternative or additional diagnoses in adults & Diagnostic test \\
\hline Intrabronchial obstruction & Bronchoscopy \\
\hline Vocal cord dysfunction & Laryngoscopy during attack \\
\hline \multirow[t]{2}{*}{ Dysfunctional breathing/panic attacks } & Blood gases during attack \\
\hline & Hyperventilation provocation test \\
\hline \multirow[t]{2}{*}{ Recurrent microaspiration } & Proximal oesophageal $\mathrm{pH}$ measurement \\
\hline & Bile salts in bronchoalveolar lavage fluid \\
\hline Cystic fibrosis (CF) & Sweat test \\
\hline Allergic bronchopulmonary aspergillosis (ABPA) & Aspergillus $\lg \mathrm{E} /$ precipitins/sputum culture \\
\hline Emphysema & High resolution CT scan \\
\hline \multicolumn{2}{|l|}{ Hypersensitivity pneumonitis } \\
\hline \multicolumn{2}{|l|}{ Bronchiectasis (including ABPA, CF) } \\
\hline Recurrent pulmonary embolism & CT pulmonary angiography \\
\hline \multicolumn{2}{|l|}{ Pulmonay arterial hypertension } \\
\hline Bronchiolitis & Transbronchial or thoracoscopic lung biopsy \\
\hline \multicolumn{2}{|l|}{ Sarcoidosis } \\
\hline \multicolumn{2}{|l|}{ Systemic } \\
\hline \multirow[t]{2}{*}{ Churg-Strauss syndrome } & Biopsy of affected organ(s) \\
\hline & Antineutrophilic cytoplasmic antibodies \\
\hline Suspected alternative or additional diagnoses in children & Diagnostic test \\
\hline \multirow{2}{*}{$\begin{array}{l}\text { Structural abnormalities (tracheobronchial malacia, } \\
\text { vascular compression/rings, tracheal stenosis/webs, cystic } \\
\text { lesions/masses, tumours/lymphadenopathy/cardiomegaly) }\end{array}$} & Fibreoptic bronchoscopy \\
\hline & Thorax CT scan \\
\hline Intrabronchial obstruction (eg, inhaled foreign body) & Rigid bronchoscopy \\
\hline Vocal cord dysfunction & History, direct observation \\
\hline Dysfunctional breathing/panic attacks & History, direct observation \\
\hline \multirow{2}{*}{$\begin{array}{l}\text { Gastro-oesophageal reflux with/without recurrent } \\
\text { microaspiration }\end{array}$} & Proximal oesophageal pH measurement \\
\hline & Bile salts in bronchoalveolar lavage fluid \\
\hline Cystic fibrosis (CF) & Sweat test \\
\hline Immune abnormalities & $\begin{array}{l}\text { Serum immunoglobulins including } \lg A, G, \\
\mathrm{M} \text { and } \mathrm{IgG} \text { subclasses and vaccine antibody } \\
\text { responses (Haemophilus, tetanus and } \\
\text { Pneumococcus) }\end{array}$ \\
\hline Bronchiectasis (including $\mathrm{CF}$, primary ciliary dyskinesia) & High resolution CT scan \\
\hline Bronchopulmonary dysplasia & History of prematurity \\
\hline \multirow[t]{2}{*}{ Bronchiolitis obliterans } & High resolution CT scan \\
\hline & Lung biopsy \\
\hline
\end{tabular}

counts and airflow limitation. ${ }^{60}$ Medical or surgical treatment of upper airway disease can improve asthma control. Patients with severe asthma should therefore be evaluated and treated for chronic rhinosinusitis, particularly if associated with nasal polyps.

\section{DEFINING 'PROBLEMATIC', 'DIFFICULT' AND 'SEVERE REFRACTORY' ASTHMA}

By excluding factors that may aggravate or complicate asthma, the subgroup with truly severe refractory asthma can be defined and distinguished from patients with 'problematic' or 'difficult' asthma.

The term 'problematic severe asthma' includes all asthma and asthma-like symptoms that remain uncontrolled despite the prescription of high-intensity asthma treatment. It is an umbrella term that comprises patients with 'difficult' asthma as well as patients with 'severe refractory' asthma.
The term 'difficult asthma' is reserved for asthma that remains uncontrolled despite the prescription of high-intensity asthma treatment due to:

- persistently poor compliance;

- psychosocial factors, dysfunctional breathing, vocal cord dysfunction;

- persistent environmental exposure to allergens or toxic substances;

- untreated or undertreated comorbidities such as chronic rhinosinusitis, reflux disease or obstructive sleep apnoea syndrome.

The term 'severe refractory asthma' should be reserved for patients with asthma in whom alternative diagnoses have been excluded, comorbidities have been treated, trigger factors have been removed (if possible) and compliance with treatment has been checked, but still have poor asthma control or frequent $(\geq 2)$ severe exacerbations per year despite the prescription of 
Table 2 Diagnosis and treatment of recognised comorbidities in severe asthma

\begin{tabular}{lll}
\hline Comorbid condition & Test & Treatment \\
\hline Gastro-oesophageal reflux & $\begin{array}{l}3 \text { months empirical therapy trial with proton } \\
\text { pump inhibitors or oesophageal pH testing }\end{array}$ & $\begin{array}{l}\text { Lifestyle modifications } \\
\text { Proton pump inhibitors } \\
\text { Surgery }\end{array}$ \\
$\begin{array}{lll}\text { Obesity with or without obstructive } \\
\text { sleep apnoea syndrome }\end{array}$ & Polysomnography & Weight control \\
& & Positive airway pressure \\
Sinus disease & & Oral appliances \\
& CT scan & Surgery \\
& Nasendocopy & Nasal irrigation with saline \\
& & Corticosteroid spray \\
Depression/anxiety & Corticosteroid drops \\
& & Surgery \\
& & Medical treatment or psychotherapy \\
\end{tabular}

high-intensity treatment or can only maintain adequate control when taking systemic corticosteroids and are thereby at risk of serious adverse effects of treatment.

For this definition, poor asthma control is defined according to Juniper et al as a score of $\geq 1.5$ by the 7-item Asthma Control Questionnaire $^{6162}$ or an equivalent score by any other standardised asthma control questionnaire. High-intensity treatment in adults is defined as $\geq 1000 \mu \mathrm{g} /$ day fluticasone equivalent and/ or daily oral corticosteroids combined with long-acting $\beta_{2}$ agonists or any other controller medication. High-intensity treatment in school age children is defined as $\geq 500 \mu \mathrm{g} /$ day fluticasone equivalent or daily oral corticosteroids combined with long-acting $\beta_{2}$ agonists or any other controller medication. High-intensity treatment in pre-school children is defined as (1) high-dose ICS and oral leukotriene receptor antagonists at the time of viral exacerbations; and/or (2) $\geq 400 \mu \mathrm{g}$ /day budesonide equivalent and oral leukotriene receptor antagonists given regularly.

\section{CLINICAL AND INFLAMMATORY PHENOTYPES OF SEVERE ASTHMA}

Although the subgroup of patients with 'severe refractory asthma' is less heterogeneous than the group of patients with 'difficult' asthma, it is far from homogeneous and may be subdivided into different phenotypes. ${ }^{37} 63$ Phenotypes have been far less well studied in children, but it is likely that those described below are part of the spectrum of asthma, at least in older children.

From a clinical point of view, three categories of patients with severe asthma seem to be of particular importance: (1) those suffering from frequent severe exacerbations with relatively stable episodes between exacerbations (exacerbation prone asthma); (2) those who develop irreversible airflow obstruction (asthma with fixed airflow obstruction); and (3) those who depend on systemic corticosteroids for daily control of their asthma (steroid-dependent asthma). ${ }^{64}$

Exacerbation-prone asthma accounts for more than $40 \%$ of severe asthma in the SARP database, ${ }^{65}$ whereas $60 \%$ of patients in the TENOR study had evidence of fixed airflow limitation. ${ }^{66}$ These latter patients are less predisposed to severe exacerbations. ${ }^{67}$ Cohort studies in children suggest that some of these patients may have failed to increase their lung function adequately and fall off their lung function centiles, ${ }^{68}$ but many adult patients with severe asthma show accelerated decline of lung function, ${ }^{69}$ particularly men with recent non-allergic asthma. ${ }^{66} 70$

A subset of patients with severe asthma requires daily systemic corticosteroids to control their asthma at the cost of serious side effects. This might be due to relative insensitivity to corticosteroids or to involvement of the paranasal sinuses and distal airways in the inflammatory process.

From a pathological point of view, at least two phenotypes of severe asthma have been proposed, each associated with distinct clinical and pathophysiological characteristics. These subtypes include the persistent eosinophilic and non-eosinophilic forms of severe asthma. ${ }^{71}$

Severe asthma with persistent eosinophilia has been put forward by Wenzel and colleagues ${ }^{71}$ and further characterised by others. $^{70-72}$ It is characterised by mixed eosinophilia and neutrophilia in bronchial biopsies and induced sputum despite the use of high-intensity ICS or oral corticosteroid treatment. This type of asthma is associated with severe exacerbations, ${ }^{8} 9$ sinus disease, ${ }^{72}$ involvement of the peripheral airways, ${ }^{71}$ airway remodelling $^{73}$ and fixed airflow obstruction, ${ }^{70}$ and responds favourably to treatment with anti-interleukin 5 monoclonal antibody. $^{9} 10$

In the non-eosinophilic subtype of severe asthma, airway eosinophils are either absent or suppressed by treatment in the presence of a high level of asthma symptoms. ${ }^{74}$ Airway inflammation in these patients with severe asthma is characterised by an increased percentage of neutrophils. ${ }^{75} 76$ The potential causal factors that induce airway neutrophilia are numerous, but it is still uncertain whether these cells play an active role in an ongoing airway damaging process.

Different phenotypes of severe asthma have also been proposed in children. ${ }^{77}$ In general, children with severe asthma have no gender bias and are highly atopic with relatively wellpreserved lung function. Subphenotyping has been mainly by inflammatory cells in induced sputum. Airway eosinophilia might be characteristic for a separate exacerbating phenotype in which food allergy is a potential factor increasing the severity of exacerbations. ${ }^{78}$

\section{TOWARDS NEW PHENOTYPES OF SEVERE REFRACTORY ASTHMA}

Clinical characterisation of patients by a single clinical characteristic or biomarker is probably not enough to describe the severe asthma phenotypes. The fact that, at a group level, clinical and pathophysiological biomarkers do not correlate strongly with one another ${ }^{79}$ suggests that they add independent information about a patient's underlying phenotype. New approaches to statistical modelling, such as cluster analysis, may enable a better definition of asthma phenotypes. The first study using factor analysis in asthma supported the idea that different dimensions of the disease-such as airway obstruction, hyper-responsiveness and 
eosinophilic inflammation-independently contribute to the disease. $^{80}$ Two other more recent studies identified different clusters of refractory asthma. ${ }^{67} 81$ The first study distinguished three clusters, one characterised by concordance between asthma symptoms and eosinophilic airway inflammation (early-onset atopic asthma) and two clusters with marked discordance between symptom expression and eosinophilic airway inflammation (obese women with symptom-predominant asthma and late-onset inflammation-predominant asthma). ${ }^{81}$ The other study identified three clusters of patients with severe refractory asthma: one cluster of obese women with late-onset non-atopic asthma, moderate reductions in $\mathrm{FEV}_{1}$ and frequent oral corticosteroid use to manage exacerbations, and two other clusters with severe airflow obstruction and bronchodilator responsiveness who differed in their ability to attain normal lung function, age of asthma onset, atopic status and use of oral corticosteroids. ${ }^{67}$

\section{U-BIOPRED}

The pan-European project Unbiased Biomarkers for the Prediction of Respiratory Disease Outcome (U-BIOPRED), ${ }^{82}$ as part of the Innovative Medicines Initiative, ${ }^{83}$ will push this further by integrating high dimensional data from invasive (bronchial biopsies), non-invasive (blood, sputum, exhaled air) and patientreported outcomes into distinct phenotype handprints by using an innovative systems biology approach. ${ }^{84}$ This will enable more detailed phenotyping of adult and paediatric severe asthma and prediction of therapeutic efficacy in view of tailored management.

\section{CONCLUSION}

Over the last 15 years there has been a lack of consensus about the definition and diagnosis of severe asthma. Research studies in patients with severe asthma have used different inclusion and exclusion criteria and the nomenclature to describe these patients has been quite confusing. There is now increasing evidence that patients with 'severe asthma' form a heterogeneous group, and that many aggravating factors may influence the clinical presentation. For the development of innovative therapies, there is an urgent need for an accurate characterisation of patients with truly severe refractory asthma and for subphenotyping these patients. The U-BIOPRED programme not only reached international consensus on the definition and diagnosis of severe asthma but, more importantly, produced for the first time a stepwise algorithm by which the patient with truly severe refractory asthma may be identified.

\section{Author footnote}

List of contributors at the U-BIOPRED International Consensus Definition and Diagnosis of Severe Asthma: Bel EH, Bergeron C, Bisgaard H, Bleecker E, Boulet L-P, Bousquet J, Brightling $\mathrm{CE}$, Bush $\mathrm{A}$, Castro M, Chanez P, Chung KF, Compton CH, Cookson W, de Boer WI, Djukanovic R, Fleming L, Gaga M, Hedlin G, Howarth PH, Ivanoff N, Kiley J, Larsson LG, Menzies-Gow A, Meyers DA, Myles D, Nething K, O'Byrne PM, Palkonen S, Polosa R, Purkins L, Rohou S, Serdrevic D, Sousa A, Sterk PJ, Ventresca G, Versnel J, Wagener AK, Wagers SS, Wenzel SE.

Funding This report was supported by grants from the European Commission Innovative Medicine Initiative Understanding Severe Asthma, IMI_Call_2008_1_12; final protocol available at http://www.imi.europa.eu/.

\section{Competing interests None.}

Contributors All authors contributed actively to the consensus meeting, revised and changed the manuscript critically and approved the version published. EHB chaired the consensus meeting, developed the framework for the review and wrote the first version of the manuscript. AS, LF, AB, KFC, JV, AHW, SSW, PJS and CC assisted in writing the manuscript and revised the subsequent versions. All participants in the consensus meeting and the U-BIOPRED consortium had the opportunity to review and revise the pre-definitive version of the manuscript. All co-authors were core members of U-BIOPRED work package "Consensus Generation".
Provenance and peer review Not commissioned; externally peer reviewed.

\section{REFERENCES}

1. Chung $\mathbf{K F}$, Godard P, Adelroth E, et al. Difficult/therapy-resistant asthma: the need for an integrated approach to define clinical phenotypes, evaluate risk factors, understand pathophysiology and find novel therapies. ERS Task Force on Difficult/ Therapy-Resistant Asthma. European Respiratory Society. Eur Respir J 1999;13:1198-208.

2. Anon. Proceedings of the ATS Workshop on refractory asthma: current understanding, recommendations, and unanswered questions. American Thoracic Society. Am J Respir Crit Care Med 2000;162:2341-51.

3. Adcock IM, Caramori G, Chung KF. New targets for drug development in asthma. Lancet 2008;372:1073-87.

4. Heaney LG, Robinson DS. Severe asthma treatment: need for characterising patients. Lancet 2005;365:974-6.

5. Holgate ST, Polosa R. The mechanisms, diagnosis, and management of severe asthma in adults. Lancet 2006;368:780-93.

6. Bush A, Saglani S. Management of severe asthma in children. Lancet 2010:376:814-25

7. Berry MA, Hargadon B, Shelley M, et al. Evidence of a role of tumor necrosis factor alpha in refractory asthma. N Engl J Med 2006;354:697-708.

8. Green RH, Brightling CE, McKenna $S$, et al. Asthma exacerbations and sputum eosinophil counts: a randomised controlled trial. Lancet 2002;360:1715-21.

9. Haldar P, Brightling CE, Hargadon B, et al. Mepolizumab and exacerbations of refractory eosinophilic asthma. N Engl J Med 2009;360:973-84.

10. Nair P, Pizzichini MM, kjarsgaard M, et al. Mepolizumab for prednisone-dependent asthma with sputum eosinophilia. N Engl J Med 2009;360:985-93.

11. Strunk RC, Bloomberg GR. Omalizumab for asthma. N Engl J Med 2006;354:2689-95

12. Castro M, Rubin AS, Laviolette M, et al. Effectiveness and safety of bronchial thermoplasty in the treatment of severe asthma: a multicenter, randomized, doubleblind, sham-controlled clinical trial. Am J Respir Crit Care Med 2009;181:116-24.

13. Ehnhage A, Olsson P, Kolbeck KG, et al. Functional endoscopic sinus surgery improved asthma symptoms as well as PEFR and olfaction in patients with nasal polyposis. Allergy 2009;64:762-9.

14. Reddy RC, Baptist AP, Fan Z, et al. The effects of bariatric surgery on asthma severity. Obes Surg 2011;21:200-6(7).

15. National Institutes of Health. National Heart, Lung, and Blood Institute. Global Initiative for Asthma. Global strategy for asthma management and prevention. NHLBI/ WHO Workshop Report. 2008. http://www.ginasthma.org/.

16. National Heart, Lung, and Blood Institute. National Asthma Education and Prevention Program. Guidelines for the diagnosis and management of asthma. http:// www.nhlbi.nih.gov/guidelines/asthma/.

17. Moore WC, Bleecker ER, Curran-Everett D, et al. Characterization of the severe asthma phenotype by the National Heart, Lung, and Blood Institute's Severe Asthma Research Program. J Allergy Clin Immunol 2007:119:405-13.

18. Anon. The ENFUMOSA cross-sectional European multicentre study of the clinical phenotype of chronic severe asthma. European Network for Understanding Mechanisms of Severe Asthma. Eur Respir J 2003;22:470-7.

19. Dolan CM, Fraher KE, Bleecker ER, et al. Design and baseline characteristics of the epidemiology and natural history of asthma: Outcomes and Treatment Regimens (TENOR) study: a large cohort of patients with severe or difficult-to-treat asthma. Ann Allergy Asthma Immunol 2004;92:32-9.

20. Chanez $\mathbf{P}$, Wenzel SE, Anderson GP, et al. Severe asthma in adults: what are the important questions? J Allergy Clin Immunol 2007:119:1337-48.

21. Brand PL, Baraldi E, Bisgaard $\mathrm{H}$, et al. Definition, assessment and treatment of wheezing disorders in preschool children: an evidence-based approach. Eur Respir $J$ 2008:32:1096-110.

22. Saglani S, Payne DN, Zhu J, et al. Early detection of airway wall remodeling and eosinophilic inflammation in preschool wheezers. Am J Respir Crit Care Med 2007; 176:858-64.

23. Maclennan C, Hutchinson P, Holdsworth $S$, et al. Airway inflammation in asymptomatic children with episodic wheeze. Pediatr Pulmonol 2006;41:577-83.

24. Bush A, Hedlin G, Carlsen $\mathrm{KH}$, et al. Severe childhood asthma: a common international approach? Lancet 2008;372:1019-21.

25. Miller MK, Johnson C, Miller DP, et al. Severity assessment in asthma: An evolving concept. J Allergy Clin Immunol 2005;116:990-5.

26. Hedlin G, Bush A, Lodrup-Carlsen K, et al. Problematic severe asthma in children: not one problem but many. A GA2LEN initiative. Eur Respir $J$ 2010;36:196-201.

27. Bousquet $\mathbf{J}$, Mantzouranis $E$, Cruz AA, et al. Uniform definition of asthma severity control, and exacerbations: Document presented for the World Health Organization Consultation on Severe Asthma. J Allergy Clin Immunol 2010;126:926-38.

28. Taylor DR, Bateman ED, Boulet LP, et al. A new perspective on concepts of asthma severity and control. Eur Respir J 2008;32:545-54.

29. FitzGerald JM, Gibson PG. Asthma exacerbations. 4: Prevention. Thorax 2006:61:992-9.

30. Reddel H, Ware S, Marks G, et al. Differences between asthma exacerbations and poor asthma control. Lancet 1999:353:364-9.

31. Robinson DS, Campbell DA, Durham SR, et al. Systematic assessment of difficult-to-treat asthma. Eur Respir J 2003;22:478-83. 
32. Bracken $\mathbf{M}$, Fleming $L$, Hall $P$, et al. The importance of nurse-led home visits in the assessment of children with problematic asthma. Arch Dis Child 2009;94:780-4.

33. Williams LK, Joseph CL, Peterson EL, et al. Patients with asthma who do not fill their inhaled corticosteroids: a study of primary nonadherence. J Allergy Clin Immunol 2007;120:1153-9

34. Williams LK, Pladevall $\mathrm{M}, \mathrm{Xi} \mathrm{H}$, et al. Relationship between adherence to inhaled corticosteroids and poor outcomes among adults with asthma. J Allergy Clin Immunol 2004;114:1288-93.

35. Horne R. Compliance, adherence, and concordance: implications for asthma treatment. Chest 2006;130(1 Suppl):65S-72S.

36. Horne R, Weinman J. Patients' beliefs about prescribed medicines and their role in adherence to treatment in chronic physical illness. J Psychosom Res 1999:47:555-67.

37. Wenzel SE. Asthma: defining of the persistent adult phenotypes. Lancet 2006;368:804-13

38. Langley SJ, Goldthorpe S, Craven M, et al. Exposure and sensitization to indoor allergens: association with lung function, bronchial reactivity, and exhaled nitric oxide measures in asthma. J Allergy Clin Immunol 2003;112:362-8.

39. Almqvist C, Wickman M, Perfetti L, et al. Worsening of asthma in children allergic to cats, after indirect exposure to cat at school. Am J Respir Crit Care Med 2001;163:694-8.

40. Denning DW, O'Driscoll BR, Powell G, et al. Randomized controlled trial of oral antifungal treatment for severe asthma with fungal sensitization: the Fungal Asthma Sensitization Trial (FAST) study. Am J Respir Crit Care Med 2009;179:11-18.

41. Ricciardolo FL, Di SA. Corticosteroid resistance in smokers with asthma. Am J Respir Crit Care Med 2004;169:1252-3.

42. Thomson NC, Chaudhuri R, Livingston E. Asthma and cigarette smoking Eur Respir J 2004:24:822-33.

43. Rayens MK, Burkhart PV, Zhang M, et al. Reduction in asthma-related emergency department visits after implementation of a smoke-free law. J Allergy Clin Immunol 2008:122:537-41.

44. Chaudhuri R, Livingston E, McMahon AD, et al. Effects of smoking cessation on lung function and airway inflammation in smokers with asthma. Am J Respir Crit Care Med 2006:174:127-33.

45. Mannino DM, Homa DM, Redd SC. Involuntary smoking and asthma severity in children: data from the Third National Health and Nutrition Examination Survey. Chest 2002;122:409-15

46. Chan-Yeung M, Malo JL. Occupational asthma. N Eng/ J Med 1995;333:107-12

47. Kogevinas $\mathbf{M}$, Anto JM, Sunyer J, et al. Occupational asthma in Europe and other industrialized areas: a population-based study. Lancet 1999;353:1750-4.

48. Salpeter S, Ormiston T, Salpeter E. Cardioselective beta-blockers for chronic obstructive pulmonary disease. Cochrane Database Syst Rev 2005;(4):CD003566.

49. Lunde H, Hedner T, Samuelsson 0, et al. Dyspnoea, asthma, and bronchospasm in relation to treatment with angiotensin converting enzyme inhibitors. BMJ 1994:308:18-21.

50. Szczeklik A, Stevenson DD. Aspirin-induced asthma: advances in pathogenesis, diagnosis, and management. J Allergy Clin Immunol 2003;111:913-21.

51. Hope AP, Woessner KA, Simon RA, et al. Rational approach to aspirin dosing during oral challenges and desensitization of patients with aspirin-exacerbated respiratory disease. J Allergy Clin Immunol 2009:123:406-10.

52. Kiljander T0, Junghard 0, Beckman 0, et al. Effect of esomeprazole $40 \mathrm{mg}$ once or twice daily on asthma: a randomized, placebo-controlled study. Am J Respir Crit Care Med 2010;181:1042-8.

53. Shore SA. Obesity and asthma: possible mechanisms. J Allergy Clin Immunol 2008;121:1087-93.

54. Harding SM, Guzzo MR, Richter JE. The prevalence of gastroesophageal reflux in asthma patients without reflux symptoms. Am J Respir Crit Care Med 2000;162:34-9.

55. Rothenberg SS, Bratton D. The effects of laparoscopic Nissen fundoplication to enhance pulmonary function in the treatment of a patient with severe asthma and gastroesophageal reflux disease. J Allergy Clin Immunol 2008:121:1069-70.

56. Macgregor AM, Rand CS. Gastric surgery in morbid obesity. Outcome in patients aged 55 years and older. Arch Surg 1993;128:1153-7.

57. Hakala K, Stenius-Aarniala B, Sovijarvi A. Effects of weight loss on peak flow variability, airways obstruction, and lung volumes in obese patients with asthma. Chest 2000;118:1315-21.
58. Stenius-Aarniala B, Poussa T, Kvarnstrom J, et al. Immediate and long term effects of weight reduction in obese people with asthma: randomised controlled study. BMJ 2000;320:827-32

59. Bresciani $\mathbf{M}$, Paradis L, Des RA, et al. Rhinosinusitis in severe asthma. J Allergy Clin Immunol 2001;107:73-80.

60. ten Brinke A, Grootendorst DC, Schmidt JT, et al. Chronic sinusitis in severe asthma is related to sputum eosinophilia. J Allergy Clin Immunol 2002;109:621-6.

61. Juniper EF, Bousquet J, Abetz L, et al. Identifying 'well-controlled' and 'not well-controlled' asthma using the Asthma Control Questionnaire. Respir Med 2006;100:616-21.

62. Reddel HK, Taylor DR, Bateman ED, et al. An official American Thoracic Society/ European Respiratory Society statement: asthma control and exacerbations: standardizing endpoints for clinical asthma trials and clinical practice. Am J Respir Crit Care Med 2009;180:59-99.

63. Bel EH. Clinical phenotypes of asthma. Curr Opin Pulm Med 2004;10:44-50.

64. Bel E, ten Brinke A. A rational approach to the management of severe refractory asthma. Treat Respir Med 2005;4:365-79.

65. Wenzel SE, Busse WW. Severe asthma: lessons from the Severe Asthma Research Program. J Allergy Clin Immunol 2007;119:14-21.

66. Lee JH, Haselkorn T, Borish L, et al. Risk factors associated with persistent airflow limitation in severe or difficult-to-treat asthma: insights from the TENOR study. Ches 2007;132:1882-9.

67. Moore WC, Meyers DA, Wenzel SE, et al. Identification of asthma phenotypes using cluster analysis in the Severe Asthma Research Program. Am J Respir Crit Care Med 2010;181:315-23.

68. Covar RA, Spahn JD, Murphy JR, et al. Progression of asthma measured by lung function in the childhood asthma management program. Am J Respir Crit Care Med 2004:170:234-41.

69. van Veen I, ten Brinke A, Sterk PJ, et al. Exhaled nitric oxide predicts lung function decline in difficult-to-treat asthma. Eur Respir J 2008;32:344-9.

70. ten Brinke A, Zwinderman AH, Sterk PJ, et al. Factors associated with persistent airflow limitation in severe asthma. Am J Respir Crit Care Med 2001;164:744-8.

71. Wenzel SE, Schwartz LB, Langmack EL, et al. Evidence that severe asthma can be divided pathologically into two inflammatory subtypes with distinct physiologic and clinical characteristics. Am J Respir Crit Care Med 1999:160:1001-8.

72. van Veen I, ten Brinke A, Gauw SA, et al. Consistency of sputum eosinophilia in difficult-to-treat asthma: a 5-year follow-up study. J Allergy Clin Immunol 2009:124:615-17.

73. Flood-Page $\mathbf{P}$, Menzies-Gow A, Phipps S, et al. Anti-IL-5 treatment reduces deposition of ECM proteins in the bronchial subepithelial basement membrane of mild atopic asthmatics. J Clin Invest 2003;112:1029-36.

74. Pavord ID, Brighting CE, Woltmann G, et al. Non-eosinophilic corticosteroid unresponsive asthma. Lancet 1999;353:2213-14.

75. Jatakanon A, Uasuf C, Maziak W, et al. Neutrophilic inflammation in severe persistent asthma. Am J Respir Crit Care Med 1999;160:1532-9.

76. Wenzel SE, Szefler SJ, Leung DY, et al. Bronchoscopic evaluation of severe asthma Persistent inflammation associated with high dose glucocorticoids. Am J Respir Crit Care Med 1997;156:737-43.

77. Bush A, Menzies-Gow A. Phenotypic differences between pediatric and adult asthma. Proc Am Thorac Soc 2009;6:712-19.

78. Bossley CJ, Saglani S, Kavanagh C, et al. Corticosteroid responsiveness and clinica characteristics in childhood difficult asthma. Eur Respir J 2009:34:1052-9.

79. Lemiere C, Ernst P, Olivenstein R, et al. Airway inflammation assessed by invasive and noninvasive means in severe asthma: eosinophilic and noneosinophilic phenotypes. J Allergy Clin Immunol 2006:118:1033-9.

80. Rosi E, Ronchi MC, Grazzini M, et al. Sputum analysis, bronchial hyperresponsiveness, and airway function in asthma: results of a factor analysis. J Allergy Clin Immunol 1999;103:232-7.

81. Haldar P, Pavord ID, Shaw DE, et al. Cluster analysis and clinical asthma phenotypes. Am J Respir Crit Care Med 2008:178:218-24.

82. Unbiased Biomarkers for the Prediction of Respiratory Disease Outcomes (U-BIOPRED). http://www.ubiopred.eu/.

83. Innovative Medicines Initiative (IMI). http://www.imi.europa.eu/.

84. Auffray C, Adcock IM, Chung F, et al. An integrative systems biology approach for understanding of pulmonary disease. Chest 2010;137:1410-16. 